\title{
1 Mutation rate of SARS-CoV-2 and emergence of mutators during
}

\section{2 experimental evolution}

3 Massimo Amicone ${ }^{1 *}$, Vítor Borges ${ }^{2 *}$, Maria João Alves ${ }^{3 *}$, Joana Isidro ${ }^{2 *}$, Líbia Zé-Zé ${ }^{3,4}$,

4 Sílvia Duarte ${ }^{5}$, Luís Vieira ${ }^{5,6}$, Raquel Guiomar7, João Paulo Gomes ${ }^{2}$, Isabel Gordo ${ }^{1 凶}$

5

$6 \quad *$ These authors contributed equally to this work.

7 IInstituto Gulbenkian de Ciência, Oeiras, Portugal.

8 2Bioinformatics Unit, Department of Infectious Diseases, National Institute of Health

9 Doutor Ricardo Jorge (INSA), Lisbon, Portugal.

$10{ }^{3}$ Centre for Vectors and Infectious Diseases Research, Department of Infectious

11 Diseases, National Institute of Health Doutor Ricardo Jorge (INSA), Águas de Moura,

12 Portugal.

13 4BioISI - Biosystems \& Integrative Sciences Institute, Faculty of Sciences, University of

14 Lisbon, Portugal.

15 5Innovation and Technology Unit, Department of Human Genetics, National Institute of

16 Health Doutor Ricardo Jorge (INSA), Lisbon, Portugal.

$17{ }^{6}$ Centre for Toxicogenomics and Human Health (ToxOmics), Genetics, Oncology and

18 Human Toxicology, Nova Medical School|Faculdade de Ciências Médicas, Universidade

19 Nova de Lisboa, Lisbon, Portugal.

$20{ }^{7}$ National Reference Laboratory for Influenza and other Respiratory Viruses,

21 Department of Infectious Diseases, National Institute of Health Doutor Ricardo Jorge

22 (INSA), Lisbon, Portugal.

${ }^{\bowtie}$ e-mail: igordo@igc.gulbenkian.pt; j.paulo.gomes@insa.min-saude.pt

Abstract: 219 words; Main text: 4618 words; Figures: 5. 


\section{Abstract}

Background and objectives. To understand how organisms evolve, it is fundamental to study how mutations emerge and establish. Here, we estimated the rate of mutation accumulation of SARS-CoV-2 in vitro and investigated the repeatability of its evolution when facing a new cell type but no immune or drug pressures.

Methodology. We performed experimental evolution with two strains of SARS-CoV-2, one carrying the originally described spike protein (CoV-2-D) and another carrying the D614G mutation that has spread worldwide (CoV-2-G). After 15 passages in Vero cells and whole genome sequencing, we characterized the spectrum and rate of the emerging mutations and looked for evidences of selection across the genomes of both strains.

Results. From the mutations accumulated, and excluding the genes with signals of selection, we estimate a spontaneous mutation rate of $1.25 \times 10^{-6} \mathrm{nt}^{-1}$ per infection cycle for both lineages of SARS-CoV-2. We further show that mutation accumulation is heterogeneous along the genome, with the spike gene accumulating mutations at rate five-fold higher than the genomic average. We also observe the emergence of mutators in the CoV-2-G background, likely linked to mutations in the RNA-dependent RNA polymerase and/or in the error-correcting exonuclease protein.

Conclusions and implications. These results provide valuable information on how spontaneous mutations emerge in SARS-CoV-2 and on how selection can shape its genome towards adaptation to new environments.

\section{Lay summary}

Mutation is the ultimate source of variation. We estimated how the SARS-COV-2 viruscause of the COVID-19 pandemic-mutates. Upon infecting cells, its genome can change at a rate of 0.04 per replication. We also find that this rate can change and that its spike protein can adapt, even within few replications.

\section{Background and objectives}

Mutation is the principal process driving the origin of genetic diversity. The mutation rate is a function of replication fidelity and represents the intrinsic rate at which genetic changes emerge prior to selection. The substitution rate, instead, is a measure of 
mutation accumulation in a given period of time and embeds the effects of selection[1]. These rates and the spectrum of the emerging mutations are fundamental parameters to understand how an organism evolves and how new variants are purged from, or establish in natural populations.

In DNA based microbes the genomic mutation rate per cell per generation, measured in laboratory conditions, is close to a constant[2]. On the other hand, for RNA viruses there is a remarkable variation in their replication fidelity[3,4]. The basic mutation rates, expressed as nucleotide substitutions per site per cell infection (s/n/c), vary between 106 to $10^{-3}$ for the several positive ssRNA viruses which have been studied[5]. Importantly, our current knowledge of the mutation rate of the human beta-coronavirus SARS-CoV-2, which is the cause of the COVID-19 pandemic[6], is based on estimates from different coronaviruses[5,7,8] and still lacks a direct quantification[9].

Laboratory evolution experiments with microbial populations allow to determine how fast mutations accumulate[10,11], and combining them with high-throughput sequencing is one of the best methods to estimate mutation rates, determine how they vary along the genome[12] and study the extent to which convergent evolution occurs $[13,14]$.

Here, via experimental evolution of two natural variants of SARS-CoV-2[15] and whole genome sequencing, we characterized the spectrum and rates of their emerging mutations, and identified specific targets of selection. Such information is important for better understanding the basic biology of this virus and to quantify how predicable the evolution of strains with different transmission capabilities can be. It may also help determining some potential genomic constraints of the virus, which are key to the design of evolution proof vaccines and antiviral drugs.

\section{Methodology}

\section{Virus growth and in vitro assay}

Vero E6 (African green monkey, Cercopithecus aethiops kidney epithelial cells, ATCC® CRL $1586^{\mathrm{TM}}$ ) cells were cultured at $37^{\circ} \mathrm{C}$ and $5 \% \mathrm{CO}_{2}$ in Minimum Essential Medium (MEM 1X, Gibco®) supplemented with 10\% fetal bovine serum (FBS), penicillin (100 units/ml) and streptomycin $(100 \mu \mathrm{g} / \mathrm{ml})+$ fungizone. The two clinical isolates Portugal/PT0054/2020 and Portugal/PT1136/2020, isolated at the National Institute of 
Health Doutor Ricardo Jorge (INSA), were used to produce the ancestors of the experimental evolution, CoV-2-D and CoV-2-G, which seeded the two laboratory evolution experiments. For this, the initial SARS-CoV-2 stock was produced by infecting Vero E6 cells (freshly grown for $24 \mathrm{~h}$ ) and incubating the cells for $72 \mathrm{~h}$. The culture supernatant was stored in aliquots at $-80^{\circ} \mathrm{C}$. The TCID 50 of viral stock was calculated according to the method of Reed and Muench[16]. All work with infectious SARS-CoV-2 strains was done inside a class III microbiological safety cabinet in a containment level 3 facility at the Centre for Vectors and Infectious Diseases Research (INSA). From the stored stocks, two 96-well plates fully inoculated with $50 \mu$ l of Vero E6 cells $\left(2.0 \times 10^{4}\right.$ cells $)$ grown for $24 \mathrm{~h}$ were infected with $50 \mu \mathrm{l}$ of the SARS-CoV-2 strains viral

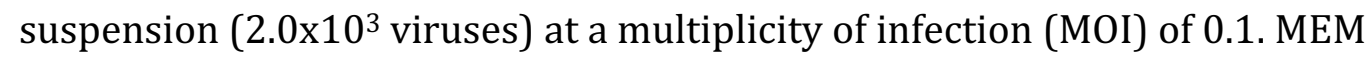
supplemented with $10 \%$ FBS, penicillin (100 units $/ \mathrm{ml})$ and streptomycin (100 $\mu \mathrm{g} / \mathrm{ml})+$ fungizone was added to each well $(50 \mu \mathrm{l})$ and the plates were incubated for $24 \mathrm{~h}$. Each well had a final volume of $150 \mu$ l. Every day, for 15 days, serial passages were done by passaging $50 \mu \mathrm{l}$ of the culture supernatant to 96-well plates (one for each SARS-CoV-2 strain under study) fully inoculated with $50 \mu$ of Vero E6 cells $\left(2.0 \times 10^{4}\right.$ cells $)$ using the same procedure and incubated in the same conditions. At day 15, total nucleic acids were extracted from $100 \mu \mathrm{l}$ of viral suspension of each well in each plate (96 samples of day $15^{\text {th }}$ for each strain) using the automated platform NUCLISENS easyMAG (Biomérieux). Confirmation of nucleic acid integrity and rough concentration estimative was made before sequencing experiment by RT-qPCR of 8 random chosen samples from each plate at day 15 (CoV-2-D and CoV-2-G) using Novel Coronavirus (2019-nCoV) RTPCR Detection Kit (Fosun Diagnostics). Samples from inoculation suspension (day 1) were also analyzed. All samples presented values of 7-10 Ct (Cycle threshold). When we infect the cells with $2 \times 10^{3}$ particle forming units (PFU), after $24 \mathrm{~h}$ the number of PFUs is around $2 \times 10^{6}$. So, assuming no major fluctuations in the viral load of the transferred suspension throughout the 15 passages and assuming a yield of approximately 1000 PFU/cell[9], the estimated number of replication cycles per passage is around 1 (i.e. $\left.\ln \left(2 \times 10^{6} / 2 \times 10^{3}\right) / \ln \left(10^{3}\right)\right)$.

\section{SARS-CoV-2 genome sequencing and bioinformatics analysis}

Genome sequencing was performed at INSA following an amplicon-based whole-genome amplification strategy using tiled, multiplexed primers[17], according to the ARTIC 
123 slight modifications, as previously described[15]. Briefly, after cDNA synthesis, whole-

124 genome amplification was performed using two separate pools of tiling primers [pools 1

125 and 2; primers version V3 (218 primers) was used for all samples:

https://github.com/artic-network/artic-

127 ncov2019/tree/master/primer schemes/nCoV-2019]. The two pools of multiplexed

128 amplicons were then pooled for each sample, followed by post PCR clean-up and Nextera

129 XT dual-indexed library preparation, according to the manufacturers' instructions.

130 Sequencing libraries were paired-end sequenced (2x150 bp) on an Illumina NextSeq 550 apparatus, as previously described[18]. Sequence read quality analysis and mapping was conducted using the bioinformatics pipeline implemented in INSaFLU

134 https://insaflu.readthedocs.io/en/latest/; as of 10 March 2021), which is a web-based

135 (and also locally installable) platform for amplicon-based next-generation sequencing 136 data analysis[18]. We performed raw reads quality analysis using FastQC v0.11.9

137 (https://www.bioinformatics.babraham.ac.uk/projects/fastqc), followed by quality improvement using

Trimmomatic

v. 0.27

139 (http://www.usadellab.org/cms/index.php?page=trimmomatic; HEADCROP:30 CROP:90 SLIDINGWINDOW:5:20 LEADING:3 TRAILING:3 MINLEN:35 TOPHRED33), with reads being conservatively cropped $30 \mathrm{bp}$ at both ends for primer clipping. Reference-based mapping was performed against the Wuhan-Hu-1/2019 reference genome sequence

143 (https://www.ncbi.nlm.nih.gov/nuccore/MN908947.3;NC_045512.2) using the BurrowWheeler Aligner (BWA_MEM) v.0.7.12 (r1039) (http://bio-bwa.sourceforge.net/)[19] integrated in multisoftware tool Snippy (https://github.com/tseemann/snippy) available in INSaFLU. The obtained median depth of coverage throughout the genome for CoV-2-D and CoV-2-G samples (except two samples excluded due to low coverage) was 4807 (IQR=3969-5242) and 5154 (IQR=4802-5439), respectively. Variant (SNP/indels) calling was performed over BAM files using LoFreq v.2.1.5 (call mode, including --callindels)[20], with indel qualities being assessed using Dindel[21]. Mutation frequency analysis was dynamic and contingent on the depth of coverage of each processed site, e.g. minor mutations at "allele" frequencies of 10\%, 2\% and 1\% (minimum cut-off used) were validated for sites with depth of coverage of at least 100-fold, 500-fold and 1000-fold, 
respectively. The median depth coverage per site for all validated mutations in CoV-2-D and CoV-2-G samples was 4219 (IQR=2508-6649) and 6424 (IQR=3076-10104), respectively. In order to assess if proximal SNPs and/or indels belong to the same mutational event (and thus, avoid overestimating the mutation rate), we identified all consecutive mutations separated by $\leq 12 \mathrm{bp}$. The mutations more likely to represent a single mutation event, i.e., those with similar frequencies (differing by $\leq 2.5 \%$ ), were further visually inspected using IGV (http://software.broadinstitute.org/software/igv/) to confirm/exclude their co-localization in the same reads. In total, this curation led to the identification $37 \mathrm{SNPs} /$ indels that were collapsed into 13 complex or multi-nucleotide polymorphisms (MNP). The effect of mutations on genes and predicted protein sequences was determined using Ensembl Variant Effect Predictor (VEP) version 103.1 (https://github.com/Ensembl/ensembl-vep; available as a self-contained Docker image)[22]. To obtain a refined annotation including all ORF1ab sub-peptides, the GFF3 genome annotation file (relative to the reference Wuhan-Hu-1/2019 genome of SARSCoV-2, acc. no. NC_045512.2) available in the coronapp COVID-19 genome annotator (http://giorgilab.unibo.it/coronannotator/)[23]was adapted to generate an annotation GTF file for input for the --gtf parameter. The parameter --distance was set to 0 . Supplementary Table 1 summarizes all mutations detected in this study and their distribution across clinical, ancestral cultures and end-point cultured lines $\left(15^{\text {th }}\right.$ passage). SARS-CoV-2 consensus sequences obtained directly from clinical samples for CoV-2-D (Portugal/PT0054/2020) and CoV-2-G (Portugal/PT1136/2020) viruses are available in GISAID under the accession numbers EPI_ISL_421457 and EPI_ISL_511683, respectively. Reads generated at the end of the experimental evolution study were deposited in the European Nucleotide Archive

(ENA)

\section{(https://www.ebi.ac.uk/ena/data/view/PRJEB43731).}

\section{Simulations of the neutral mutation accumulation}

To obtain a non-equilibrium neutral expectation of the site frequency spectrum of mutations, we performed forward-simulations to model mutation accumulation using the mutation rate inferred from the experiment. We model an organism with a bi-allelic genome of size $\mathrm{L}=30000$ ( $\sim$ SARS-CoV-2). An initially isogenic population undergoes 15 cycles of growth, mutation and bottleneck, according to the following life cycle:

1. A clonal population starts with an inoculum size of 2000 . 
2. Each genome replicates $X$ times. We assume the burst size $X$ to be Poisson distributed with mean 1000.

3. For each of the replicating genomes we introduce a Poisson number of mutations with mean 0.1 (corresponding to a rate of $3.3 \times 10^{-6} \mathrm{nt}^{-1}$ cycle $^{-1}$ ). We assume mutations to emerge with uniform probability in the parental genome and we allow for back-mutation.

4. After replication and mutation, we sample $1 / 1000$ of the individual genomes.

5. Repeat steps 2-4, 15 times.

We validated the simulation code by confirming expected outcomes: mutations accumulate linearly over time and the posterior estimation of the mutation rate retrieves the original value (bottom plot in Fig. S3b).

After 15 cycles we collect the artificial genomes from 100 independent simulations, and compute their site frequency spectrum as in the experiment. In order to test whether cross-well contamination could justify the observed site frequency distribution, we modified the previous algorithm by introducing migration. At each cycle $t$, after each bottleneck event, a fraction of viral genomes $(m=0.1)$ is replaced by migrants sampled from a pool of genomes that have undergone $t$ cycles of growth. The algorithm was written in R (version 3.6.1) and the results analyzed in RStudio[24].

\section{Mutation accumulation rates in all, synonymous and non-synonymous sites}

To quantify the rate at which mutations accumulate during the experiment, we compute $M_{a}(r)=\frac{\sum f_{r}}{P * L_{r}}$, where $f_{r}$ is the frequency of all mutations observed in region $r, \mathrm{P}=15$ the number of passages and $L_{r}$ the length of region $r$. For the genome-wise mutation accumulation $L_{r}=29903$, the entire genome of SARS-CoV-2. We also computed the mutation accumulation rates at synonymous and non-synonymous sites. In these cases, the synonymous rate is given by $M_{a}(r, s y n)=\frac{\sum f_{r}}{P * L_{r} * p_{r, s y n}}$, where $p_{r, s y n}$ is the proportion of mutations in region $r$, leading to synonymous changes. Equivalently the nonsynonymous rate is $M_{a}\left(r, n_{-} s y n\right)=\frac{\sum f_{r}}{P * L_{r} * p_{r, n_{-} s y n}}$. In practice, assume the region of interest has sequence $r$ : ATGTTT. For each base we count the proportion of mutations that would change (or not) the corresponding amino acid. In the example $p_{r, n_{-} s y n}=3 / 3+3 / 3+$ $3 / 3+3 / 3+3 / 3+2 / 3=17 / 18,17$ mutations out of the possible 18 are non- 
synonymous and only one is synonymous (ATGTTC). Therefore, in this example, the total size is $L_{r}=6, p_{r, n_{s} s y n}=17 / 18$ and $p_{r, s y n}=1 / 18$. Following this method, we calculated the genome-wise and gene-specific mutation accumulation rates in all, synonymous or non-synonymous sites (Fig. 1-4 and Fig. S4-5). The genomic sequences of each region

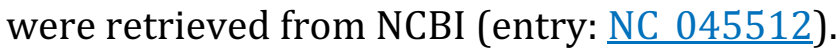

\section{$\mathrm{pN} / \mathrm{pS}$ calculation and confidence interval}

Within a given region $r$, we computed $p N(r)$ as the summed frequencies of all the observed non-synonymous mutations over the number of all possible non-synonymous changes in that region: $p N(r)=\frac{\sum f_{r}}{N_{r, n_{s} s y n}}$, where $N_{r, s y n}=3 L_{r} p_{r, n_{s y n}}$. Equivalently, we computed the synonymous counterpart: $p S(r)=\frac{\sum f_{r}}{N_{r, s y n}}$. In the previous example, within the region $r$ : ATGTTT, $N_{r, n \_s y n}=17$ while $N_{r, \text { syn }}=1$. Finally, the $p N / p S$ statistics is the ratio of $p N$ and $p S$ and its expected value is 1 under neutrality. To test the deviation from 1 , we report the $p N / p S$ together with its confidence interval. Being the $p N / p S$ a ratio of proportions, we computed the $95 \%$ confidence intervals of risk ratios, specifically as

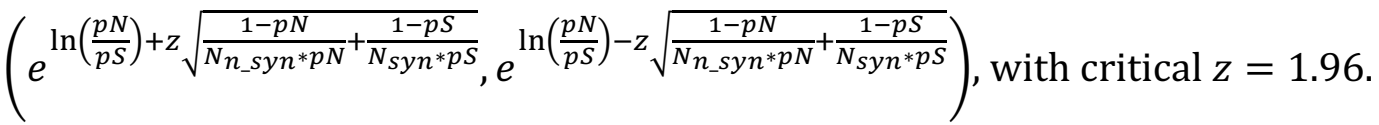

\section{Results}

\section{Experimental evolution design and ancestor backgrounds.}

Two SARS-CoV-2 viral strains were isolated from two non-related patients for continuous propagation in Vero cells (see Methodology, Fig. 1a). These were chosen according to their polymorphism at amino acid position 614 of the spike protein: CoV-2-D carries a D and CoV-2-G carries a derived mutation which changes the D into a G. This D614G mutation in spike emerged early in the pandemic, increased the infectivity of the virus and became prevalent worldwide[25]. Here, we want to test for differences in their mutation rates, spectrum and/or in the selective forces as the strains are propagated in cells.

In order to discriminate de novo mutations from standing genetic variation, we identified the mutations (relative to the Wuhan-Hu-1/2019 reference genome sequence, Wu et al., 2020) that were already present at the start of our evolution experiment (see the list and their frequencies in Fig. S1). 
If the mutation rate is similar to that of the mouse hepatitis virus (MHV) or that of the SARS-CoV (about 3.5 $\times 10^{-6}$ and $2.5 \times 10^{-6} \mathrm{nt}^{-1}$ cycle $^{-1}$, respectively)[5,7] hundreds of mutations should accumulate, many of which are expected to be neutral but some could reflect adaptation to the experimental conditions.

\section{Mutation accumulation and spectrum after 15 passages of SARS-CoV-2 evolution.}

We considered de novo mutations those that reached a frequency of at least $1 \%$, supported by a minimum of 10 reads and that were not detected in either the ancestor or the original clinical isolate from which the ancestor was derived (full list in Supplementary Table 1). Propagation of the $96 \mathrm{CoV}-2$-D derived lines resulted in 1753 de novo mutations, while the 96 lines derived from CoV-2-G resulted in 6181 de novo mutations ( $\mathrm{n}=94$ as in two lines the sequencing had poor coverage) (Fig. 1b). The much higher number of mutations in the CoV-2-G background, compared to CoV-2-D, is explained by 15 of these lines where many more mutations were observed (Fig. 1b). These lines, hereafter referred to as mutators, are characterized by a larger proportion of SNPs compared to the non-mutator lines where, instead, deletions account for more than $20 \%$ of all de novo mutations (Fig. 1c).

The frequency of mutator clones was estimated to be between 1 and $2 \%$ after 15 infection cycles, since these were the frequencies measured for the vast majority of mutations observed in the mutator lines. The genetic cause of the mutator phenotype is difficult to determine but it could likely be hidden within the mutations that occurred in the RNAdependent RNA polymerase (Nsp12) and/or in the error-correcting exonuclease protein (Nsp14)[8]. Indeed, looking at the mutations that are specific to the lineages with mutators, we found 8 non-synonymous mutations in Nsp12 (one leading to a stop at amino-acid 670) and 9 non-synonymous mutations in Nsp14 (one leading to a stop at amino acid 78) (Supplementary Tables 2-3). Any of these mutations could potentially lead to the observed change in mutation rate, but none of these have been associated with an increased mutational load of the circulating viruses[27].

Next, we obtained the per-base per-passage rate at which mutations accumulated $\left(\mathrm{Ma}_{\mathrm{a}}\right)$, from the frequencies of the observed mutations. As a $24 \mathrm{~h}$ passage in our experiment corresponds to $\sim 1$ cell replication cycle (see Methodology), we hereafter report such rate of mutation accumulation per unit of replication cycle $\left(\mathrm{nt}^{-1} \mathrm{cycle}^{-1}\right)$. Interestingly, the nonmutator lines of CoV-2-G show a significantly lower accumulation rate compared to the 
CoV-2-D lines $\left(\mathrm{P}<10^{-6}\right.$, Two-sample Kolmogorov-Smirnov test) (Fig. 1d). However, this difference between the two backgrounds is more likely due to differences in selection rather than differences in mutation rates, as we will explain later on. The SNPs accumulated over 15 passages show that both genomic backgrounds have a strong propensity to accumulate C-> T mutations (Fig. 1e), a well-known bias of SARSCoV-2[28]. In the mutator lines, the main mutation bias changed from C->T to G->T (Fig. 1e), also observed in SARS-CoV-2 samples collected during the recent COVID-19 pandemic $[29,30]$.

It is important to notice that, both the accumulation of mutations and the biases we observe in the data (Fig. 1b-e) might have been shaped by selection and deviate from a neutral rate and spectrum of mutations. In fact, on one hand positive selection can increase the frequencies of beneficial mutations and on the other hand purifying selection can purge the deleterious alleles. Therefore, we next looked for evidences of selection in the mutation accumulation data.

\section{Signs of selection: Site frequency spectrum and heterogeneity across genes.}

In serial propagation experiments with SARS-CoV-2, it is extremely difficult to pick a single virus[31]. In our experiment the effective population size is considerably large (see Methodology), and thus could be insufficient to remove the effects of either positive or negative selection[10]. Indeed, several patterns in the data indicate that selection played a significant role in the experimentally evolved SARS-CoV-2 lines.

The distribution of allele frequencies in a sample, i.e. the site frequency spectrum, has a well-known theoretical expectation under a simple equilibrium neutral model of molecular evolution (Chap. 5 pg. 233 of B. Charlesworth \& D. Charlesworth., 2010). But, this distribution is sensitive to the action of selection and also to complex demographic events, such as population bottlenecks. Given the bottlenecks occurring in our experiments and the slow evolutionary time elapsed during the 15 infection cycles, the neutral theoretical expectation at equilibrium may not apply. To obtain a nonequilibrium expectation of the site frequency spectrum, we performed forwardsimulations (see Methodology). We assumed that neutral mutations occur at a rate of $3.3 \times 10^{-6} \mathrm{nt}^{-1} \mathrm{cycle}^{-1}$, similar to that estimated from the data, and simulated populations evolving under neutrality. The site frequency spectrum of the mutations accumulated in both $\mathrm{CoV}-2-\mathrm{D}$ or $\mathrm{CoV}-2-\mathrm{G}$ lines deviates significantly from the neutral expectation 
predicted by the simulations (Fig. 2a). High frequency mutations are not expected under neutrality (mutations with frequencies above 30\% are reported in Fig. S2). To test whether possible contamination among wells could explain the observed site frequency spectrum, we performed additional simulations with migration (see Methodology). Even when considering a migration rate of $10 \%$, the neutral site frequency spectrum is still incompatible with the experimental data (Fig. S3a). Furthermore, the $10 \%$ migration between wells should not significantly change the estimation of mutation rate (Fig. S3b). Thus, the data strongly suggest that positive selection has increased the frequency of beneficial mutations and skewed the spectrum of the mutations (Fig. 2a).

A second evidence of selection comes from the considerable variation in the rate of mutation accumulation observed across the SARS-CoV-2 genome (Fig. 2b, Fig. S4). When excluding the mutator lines, the $S$ gene, which codes for the spike protein, has the highest rate of mutation accumulation among the different genes (Fig. 2b). Remarkably, the spike accumulated $13.5 \pm 0.4 \times 10^{-6} \mathrm{nt}^{-1} / \mathrm{cycle}^{-1}$ mutations in the CoV-2-G genotype (excluding mutators), and $17.1 \pm 1.0 \times 10^{-6}$ in the CoV-2-D genotype, about five-fold the corresponding genomic averages, suggesting the strong action of positive selection. In the mutator lines, the spike gene evolved $\sim 2$ times faster than the non-mutators (Fig. S4). This observation is in contrast with the expectation of a constant increase in mutation rate across the genome and suggests that more complex selective forces might be acting on the mutator phenotype (see the heterogeneity of the mutation rate across the CoV-2G mutator genome in Fig. S4). Overall, the data confirmed that selection has shaped the way mutations accumulated. Therefore, in order to obtain a more accurate quantification of the spontaneous rate of mutation, we performed a more systematic analysis of the sites under selection.

\section{Identifying regions under selection}

From the frequencies of all mutations observed in the CoV-2-D and CoV-2-G non-mutator lines, we computed an accumulation rate of $3.7 \times 10^{-6}$ and $2.9 \times 10^{-6} \mathrm{nt}^{-1} \mathrm{cycle}^{-1}$, respectively (Fig. 1d). Given that during our experiment, selection affected the allele frequencies (Fig. 2), such rates may deviate from the spontaneous mutation rates of the virus. In order to attempt to estimate the spontaneous mutation rate we first focused on synonymous mutations, which, if neutral, should accumulate at the rate at which they occur[33]. Focusing on the synonymous changes, we estimated a basic mutation rate of $3.8 \times 10^{-6} \mathrm{nt}$ 
${ }^{1}$ cycle $^{-1}$ for the CoV-2-D background and $1.2 \times 10^{-6} \mathrm{nt}^{-1}$ cycle $^{-1}$ for the CoV-2-G (Fig. S5a). However, the rate of non-synonymous mutation in CoV-2-D is lower than the synonymous one (Fig. S5a-b), suggesting the action of purifying selection on nonsynonymous sites or positive selection on the synonymous sites, leading to their increase in frequency[34,35]. To distinguish between the two cases, we compared the accumulation rate of synonymous mutations in the entire genome $\left(M_{a}^{S y n}\right)$, with the accumulation rate of synonymous mutations excluding one gene at the time $\left(M_{a}^{S y n, \Delta g}\right)$. This approach revealed that a remarkable accumulation of synonymous mutations in the Nsp6 gene led to the overestimation of the mutation rate in the CoV-2-D background (Fig. S5c). In contrast, for the CoV-2-G background this approach indicates that the estimation of $M_{a}^{S y n}=1.2 \times 10^{-6} \mathrm{nt}^{-1}$ cycle $^{-1}$ is homogeneous across the genome and can provide a first estimation of its mutation rate (Fig. S5d).

Since the synonymous mutations alone could not provide a correct estimation of mutation rate, we followed a different approach: identify the regions under selection in either the CoV-2-D or CoV-2-G lines and exclude them from the estimation of the spontaneous mutation rate. First, we compared the relative accumulation of nonsynonymous and synonymous mutations, via the $p N / p S$ statistics (equivalent of $d N / d S$ for polymorphic samples, see Methodology). In the CoV-2-D background, the $p N / p S$ of the $S$ and the Nsp6 genes significantly differ from 1 (Fig. 3a). The spike accumulated more non-synonymous mutations consistent with the action of positive selection $(p N / p S=4.4$, 95\% confidence interval: [1.4,13.9]), while the Nsp6 accumulated more synonymous mutations, consistent with our previous findings $(p N / p S=0.02,95 \%$ confidence interval: $[0.00,0.12]$, Fig. S5c). In particular, the synonymous change A11041G was found in 88 evolved populations (out of 96), but also at frequency below our 1\% threshold in the ancestral population, suggesting that such mutation was incorrectly considered as de novo and that the estimated mutation accumulation in Nsp6 was the resulting artifact.

373 Due to the limited number of mutations within each gene and the fact that we are comparing evolving populations (rather than divergent species), the $p N / p S$ may lack the power to identify additional regions under selection[36]. To overcome this issue and to identify additional genes affecting the estimation of the mutation rate, we computed the rate of mutation accumulation excluding one gene at the time and compared this with the entire genome (see Methodology and Fig. 3c-d). With this outlier-detecting method we confirmed that the S and the Nsp6 genes affected the estimation of mutation rate in the 
CoV-2-D strain, we could observe that the $\mathrm{S}$ gene is likely under selection also in the CoV2-G strain, and we identified Nsp3 as an additional region with a different rate of mutation accumulation (Fig. 3c-d). In particular, Nsp3 accumulated fewer mutations than the genomic average in both CoV-2-D and CoV-2-G strains, suggesting the action of purifying selection (Fig. 3c-d).

Overall, we conclude that during our experiment, the spike protein was under strong selection in both backgrounds, but also other genes biased the estimation of mutation rate.

\section{Estimation of mutation rates and bias excluding genes with signs of selection}

Non-neutral processes have shaped the allele dynamics in our experiment. To get a more realistic estimate of the mutation rate prior to selection, we excluded from the analysis the Nsp3, Nsp6 and S genes, which have shown signs of selection in at least one of the two backgrounds (Fig. 3). By doing this, we estimate a spontaneous mutation rate of $1.3 \pm 0.2 \times 10^{-6} \mathrm{nt}^{-1}$ cycle $^{-1}$ for the CoV-2-D background and $1.2 \pm 0.2 \times 10^{-6} \mathrm{nt}^{-1}$ cycle $^{-1}$ for the CoV-2-G (excluding mutators) (Fig. 4a). The estimated mutation rate is similar across backgrounds, suggesting that the previously observed differences were due to selection (Fig. 1d and S5). Importantly, the estimated mutation rate of CoV-2-G is consistent with that obtained using the synonymous mutations only (see Fig. S5a) and the estimated mutation rate of CoV-2-D is consistent with that obtained using the synonymous mutations only and excluding the Nsp6 (see Fig. S5c). We quantified again the relative proportion of single nucleotide changes and confirmed that both backgrounds have a spontaneous bias towards $\mathrm{C}>\mathrm{T}$ mutations and the mutator changes this bias towards $\mathrm{G}>\mathrm{T}$ mutations (Fig. 4b).

Excluding the genes with signs of selection was our best attempt at quantifying the spontaneous mutation rate of SARS-CoV-2. However, it is important to note that this may still underestimate the real one due to the fact that we ignored mutations with a frequency below the $1 \%$ threshold.

\section{Convergent targets of selection on Spike}

The spike protein showed clear signs of adaptation during our evolution experiment, so we next focused on the specific sites under selection and compared them with the new spike variants that spread in the human population. We first quantified the level of 
convergence at the nucleotide and amino levels between CoV-2-D and CoV-2-G. We note that convergence between the two backgrounds reflects true independent origin of the mutations, as they were propagated and processed for sequencing independently. In contrast, convergence within replicates of the same background could also result from some possible cross-contamination or from undetected standing variation. At the amino level, 20 specific sites and 3 regions were hit independently in both backgrounds (Fig. 5a, Supplementary Table 4). We find high evolutionary convergence at the S1/S2 cleavage site: three distinct deletions (675-QTQTN-679 del; 679-NSPRRAR-685 del and 679-NSPRRARSVA-688) emerge multiple times in both backgrounds. Such changes have been previously shown to emerge rapidly in Vero cells and to be important for the virus cell tropism[37]. Apart from these deletions, mutations of the Arginine 682 were also highly convergent, most likely because they trigger a similar functional effect, i.e., knock out of the furin cleavage site[38]. Notably, another deletion in this region (678TNSPRRARS-686 del) was frequently observed, still it was exclusive of CoV-2-D lines ( $n=$ 58), suggesting that the conformation changes mediated by D614G may influence the directionality of the evolution towards the knock out of the furin-cleavage site[39].

Some level of evolutionary convergence could also be found for the structural genes N, E and $M$ suggesting that adaptation could also have occurred in these genes (Supplementary Table 5).

The inferred mutators in the CoV-2-G background also carry many mutations in the spike protein including in the receptor binding domain -RBD- (amino acid changes at positions $328,339,364,416,454,465,474,479,482,522$ and 524) and multi cleavage site regions (positions 798 and 799) (Fig. 5b).

When scrutinizing the list of non-synonymous mutations in the spike that emerged during our experiment in both backgrounds or in the mutator lines, we found 24 aminoacid changes that were also observed in the natural population of SARS-CoV-2 (until the 24th of October 2021; https://nextstrain.org/ncov/gisaid/global) (full list reported in Supplementary Table 6 and highlighted in bold in Fig. 5). Among these, we observed the mutations H655Y (present in the variant of concern Gamma, lineage P.1, originated in Brazil), D215G (present in the variant of concern Beta, lineage B.1.351, firstly identified in South Africa) and D253G (found in lineage B.1.426, mostly detected in the US) (Fig. 5b)[40]. 


\section{Conclusions and implications}

446 The SARS-CoV-2 beta-coronavirus, first observed in the Wuhan province of China[6], has

447 infected at least 246 million people causing more than a 5 million toll of deaths in the

448 human population (as of 2 November 2021; https://covid19.who.int/). Since it was first

449 sequenced[26] the virus has been accumulating 0.44 substitutions per week at close to

450 linear rate. Here we estimate its rate of spontaneous mutation to be of the order of $10^{-6}$

451 per base per cell infection, consistent with previous estimations in other

452 coronaviruses[9]. New beneficial mutations did spread to high frequencies and

453 considerable convergent evolution was detected across different genomic backgrounds.

454 We also observe viral populations with an increased mutation rate emerging just within

45515 days of propagation in cells. This suggests that the mutation rate of SARS-CoV-2 can

456 increase without significant loss of viability (at least in the short run) and that strategies

457 to reduce viral fitness using mutagens should be tested with precaution[41,42].

458 Overall the results show the remarkable ability of SARS-CoV-2 to adapt to new 459 environments, in particular via convergent evolution of its spike protein in cells, and is 460 fully consistent to its rapid adaptation to different hosts[43,44].

461

462 Data and materials availability

463 SARS-CoV-2 consensus genome sequences obtained directly from clinical samples for 464 CoV-2-D (Portugal/PT0054/2020) and CoV-2-G (Portugal/PT1136/2020) viruses are 465 available in GISAID under the accession numbers EPI_ISL_421457 and EPI_ISL_511683, 466 respectively. Reads generated throughout the experimental evolution in this study were 467 deposited in the European Nucleotide Archive

(ENA)

468 (https://www.ebi.ac.uk/ena/data/view/PRJEB43731). The code for the neutral model 469 was deposited on https://github.com/AmiconeM/neutralviralpassage.

470

471 Acknowledgments: We would like to thank the personnel of the IGC Genomic Facility 472 for their assistance. 
474 Funding: M.A. was supported by “Fundação para a Ciência e Tecnologia” (FCT),

475 fellowships PD/BD/138735/2018, respectively. Research was supported by FCT

476 Project PTDC/BIA-EVL/31528/2017 to I.G. and by funds from Portuguese NIH.

477

478 Author contributions: IG, MJA and JPG designed the project. MJA and LZZ performed the culture and RNA extraction experiments. VB and JI performed the pre-sequencing wetlab procedures, bioinformatic analysis and data analysis. SD and LV performed and supervised the wet-lab sequencing procedures. IG, MA performed the data analysis and the simulations. MJA, LV and JPG provided materials and reagents. IG wrote the initial draft of manuscript. VB, MJA, MA and JI contributed equally to this work. All authors contributed in the final writing of the manuscript and gave final approval for publication.

Ethical statement: The Portuguese NIH is authorized by the Portuguese Authorities'

(General-Directorate of Health and the Authority for Working Conditions) to handle and propagate Risk group 2 and 3 microorganisms. All culture procedures were performed inside a class III microbiological safety cabinet in a containment level 3 facility. This study is covered by the ethical approval issued by the Ethical Committee ("Comissão de Ética para a Saúde") of the Portuguese National Institute of Health.

Competing interests: The authors declare no competing interests.

Additional information: Supplementary information is available for this paper. 496 Correspondence and requests for materials should be addressed to igordo@igc.gulbenkian.pt; j.paulo.gomes@insa.min-saude.pt. 


\section{References}

1. Lin JJ, Bhattacharjee MJ, Yu CP et al. Many human RNA viruses show extraordinarily

501 stringent selective constraints on protein evolution. Proc Natl Acad Sci U S A 2019, DOI:

502 10.1073/pnas.1907626116.

503

2. Drake JW. A constant rate of spontaneous mutation in DNA-based microbes. Proc Natl

504 Acad Sci U S A 1991, DOI: 10.1073/pnas.88.16.7160.

505 3. Drake JW. Rates of spontaneous mutation among RNA viruses. Proc Natl Acad Sci U S A 1993, DOI: 10.1073/pnas.90.9.4171.

507

4. Drake JW, Charlesworth B, Charlesworth D et al. Rates of spontaneous mutation.

508 Genetics 1998, DOI: 10.1093/genetics/148.4.1667.

509 5. Sanjuán R, Nebot MR, Chirico N et al. Viral Mutation Rates. J Virol 2010, DOI:

$510 \quad$ 10.1128/jvi.00694-10.

511 6. Zhu N, Zhang D, Wang W et al. A Novel Coronavirus from Patients with Pneumonia in

512 China, 2019. N Engl J Med 2020, DOI: 10.1056/nejmoa2001017.

513 7. Eckerle LD, Lu X, Sperry SM et al. High Fidelity of Murine Hepatitis Virus Replication

514 Is Decreased in nsp14 Exoribonuclease Mutants. J Virol 2007;81:12135-44.

515 8. Eckerle LD, Becker MM, Halpin RA et al. Infidelity of SARS-CoV Nsp14-exonuclease

516 mutant virus replication is revealed by complete genome sequencing. PLoS Pathog

$517 \quad 2010 ; 6: 1-15$.

518 9. Bar-On YM, Flamholz A, Phillips R et al. Sars-cov-2 (Covid-19) by the numbers. Elife 519 2020, DOI: 10.7554/eLife.57309.

520 10. Chao L. Fitness of RNA virus decreased by Muller's ratchet. Nature 1990, DOI: $10.1038 / 348454 a 0$.

522 11. Duarte E, Clarke D, Moya A et al. Rapid fitness losses in mammalian RNA virus clones 523 due to Muller's ratchet. Proc Natl Acad Sci U S A 1992, DOI: 10.1073/pnas.89.13.6015. 

mutation rate. Nat Rev Genet 2016, DOI: 10.1038/nrg.2016.104.

13. Bull JJ, Badgett MR, Wichman HA et al. Exceptional convergent evolution in a virus. Genetics 1997, DOI: 10.1093/genetics/147.4.1497. convergence. Science (80- ) 2012, DOI: 10.1126/science.1212986.

Spike Y839 variant in Portugal. Emerg Microbes Infect 2020, DOI:

16. Reed LJ, Muench H. A simple method of estimating fifty per cent endpoints. Am J Epidemiol 1938, DOI: 10.1093/oxfordjournals.aje.a118408.

538 18. Borges V, Pinheiro M, Pechirra P et al. INSaFLU: An automated open web-based 539 bioinformatics suite "from-reads" for influenza whole-genome-sequencing-based 540 surveillance. Genome Med 2018, DOI: 10.1186/s13073-018-0555-0.

541 19. Li H, Durbin R. Fast and accurate short read alignment with Burrows-Wheeler transform. Bioinformatics 2009, DOI: 10.1093/bioinformatics/btp324.

543 20. Wilm A, Aw PPK, Bertrand D et al. LoFreq: A sequence-quality aware, ultra-sensitive

544 variant caller for uncovering cell-population heterogeneity from high-throughput

545 sequencing datasets. Nucleic Acids Res 2012, DOI: 10.1093/nar/gks918.

546 21. Albers CA, Lunter G, MacArthur DG et al. Dindel: Accurate indel calls from short-read 547 data. Genome Res 2011, DOI: 10.1101/gr.112326.110.

548 22. McLaren W, Gil L, Hunt SE et al. The Ensembl Variant Effect Predictor. Genome Biol 
2016, DOI: 10.1186/s13059-016-0974-4.

23. Mercatelli D, Triboli L, Fornasari E et al. Coronapp: A web application to annotate and monitor SARS-CoV-2 mutations. J Med Virol 2021;93:3238-45. Comput 2019.

25. Korber B, Fischer WM, Gnanakaran S et al. Tracking Changes in SARS-CoV-2 Spike: Evidence that D614G Increases Infectivity of the COVID-19 Virus. Cell 2020, DOI: 10.1016/j.cell.2020.06.043.

26. Wu F, Zhao S, Yu B et al. A new coronavirus associated with human respiratory disease in China. Nature 2020, DOI: 10.1038/s41586-020-2008-3. association with increased genome-wide mutation load. PeerJ 2020, DOI: 10.7717/peerj.10181.

28. Matyášek R, Kovařík A. Mutation patterns of human SARS-CoV-2 and bat RATG13 coronavirus genomes are strongly biased towards $\mathrm{C}>\mathrm{U}$ transitions, indicating rapid evolution in their hosts. Genes (Basel) 2020, DOI: 10.3390/genes11070761. pandemic. Exp Mol Med 2021;53:1229-37. diversity in SARS-COV-2. Elife 2021, DOI: 10.7554/eLife.66857. 10.1371/journal.ppat.1009929.

572 32. JIGGINS C. Elements of Evolutionary Genetics. B. Charlesworth \& D. Charlesworth. 573 Roberts \& Company. 2010. 768 pages. ISBN 9780981519425. Price $\$ 80$ (hardback). 
Genet Res (Camb) 2010, DOI: 10.1017/s001667231000042x.

575

576

577

578

579

580

581

582

583

584

585

586

587

588

589

590

591

592

593

594

595

596

597

598

33. Kimura M. Evolutionary rate at the molecular level. Nature 1968, DOI:

10.1038/217624a0.

34. Novella IS, Zárate S, Metzgar D et al. Positive selection of synonymous mutations in vesicular stomatitis virus. J Mol Biol 2004, DOI: 10.1016/j.jmb.2004.08.003.

35. Zanini F, Neher RA. Quantifying Selection against Synonymous Mutations in HIV-1 env Evolution .J Virol 2013, DOI: 10.1128/jvi.01529-13.

36. Kryazhimskiy S, Plotkin JB. The population genetics of dN/dS. PLoS Genet 2008, DOI: 10.1371/journal.pgen.1000304.

37. Sasaki M, Uemura K, Sato A et al. SARS-CoV-2 variants with mutations at the S1/ S2 cleavage site are generated in vitro during propagation in TMPRSS2-deficient cells. PLoS Pathog 2021;17:1-17.

38. Liu Z, Zheng H, Lin H et al. Identification of Common Deletions in the Spike Protein of Severe Acute Respiratory Syndrome Coronavirus 2. J Virol 2020, DOI: 10.1128/jvi.00790-20.

39. Gobeil SMC, Janowska K, McDowell S et al. D614G Mutation Alters SARS-CoV-2 Spike Conformation and Enhances Protease Cleavage at the S1/S2 Junction. Cell Rep 2021, DOI: 10.1016/j.celrep.2020.108630.

40. Makoni M. South Africa responds to new SARS-CoV-2 variant. Lancet (London, England) 2021, DOI: 10.1016/S0140-6736(21)00144-6.

41. Jensen JD, Stikeleather RA, Kowalik TF et al. Imposed mutational meltdown as an antiviral strategy. Evolution (N Y) 2020, DOI: 10.1111/evo.14107.

42. Jensen JD, Lynch M. Considering mutational meltdown as a potential SARS-CoV-2 treatment strategy. Heredity (Edinb) 2020, DOI: 10.1038/s41437-020-0314-z.

43. Gu H, Chen Q, Yang G et al. Adaptation of SARS-CoV-2 in BALB/c mice for testing 
bioRxiv preprint doi: https://doi.org/10.1101/2021.05.19.444774; this version posted November $24,2021$. The copyright holder for this preprint (which was not certified by peer review) is the author/funder, who has granted bioRxiv a license to display the preprint in perpetuity. It is made available under aCC-BY-NC-ND 4.0 International license.

600 44. Tegally H, Wilkinson E, Giovanetti M et al. Emergence of a SARS-CoV-2 variant of

601 concern with mutations in spike glycoprotein. Nature 2021, DOI: 10.1038/s41586-021-

$602 \quad 03402-9$.

603

604 Figures

a

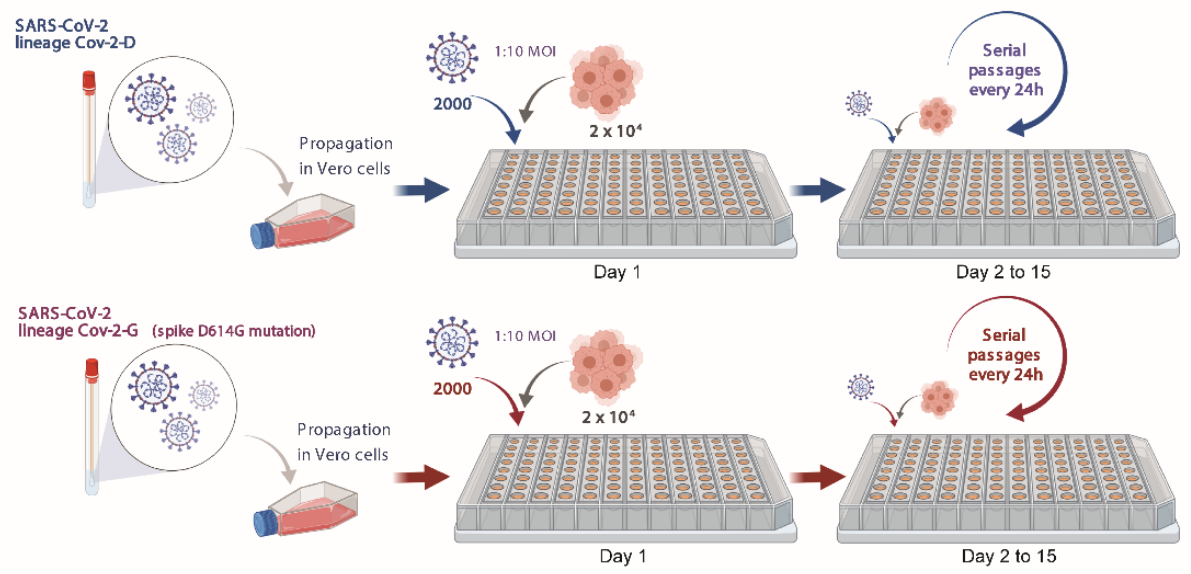

b Background CoV-2-D

CoV-2-G CoV-2-G (mutators) c
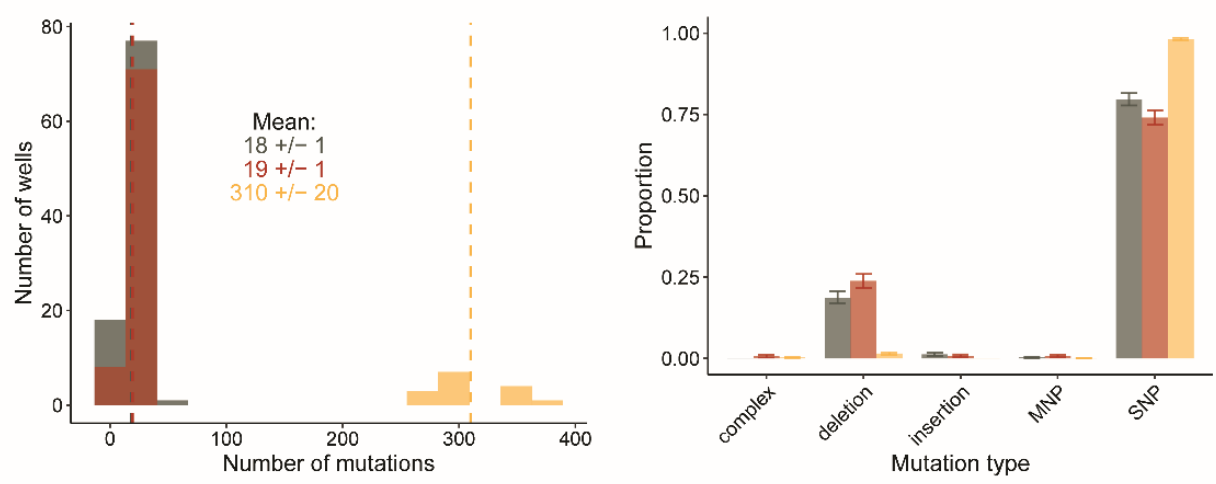

d
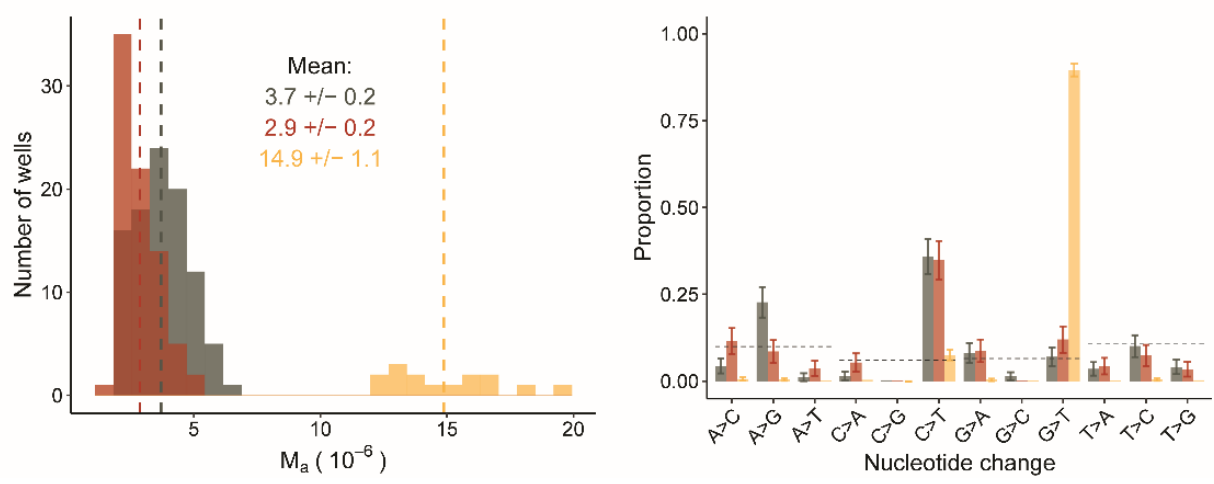
606 Fig. 1| Experimental design and mutation accumulation after 15 passages of SARS-

607 CoV-2 evolution. a, Schematic of the experimental design of the mutation accumulation

608 experiments where two viral backgrounds were propagated in Vero cells (figure created

609 with BioRender.com). b, Number of mutations observed in each well and group. 15 lines of

610 the CoV-2-G background accumulated a larger number of mutations and thus were defined

611 as mutators (gold). The means of each group are presented by vertical dashed lines and

612 reported in the figure (+/- 2SEM). c, Proportion of mutation types in each group. Complex

613 mutations and multi-nucleotide polymorphisms (MNP) are defined in the Methodology. d,

614 Mutation accumulation per base per infection cycle $\left(M_{a}\right)$ was calculated by summing the

615 observed mutation frequencies as: $M_{a}=\frac{\sum f}{P * G}$, where $P$ is the number of passages $(P=15)$

616 and $\mathrm{G}$ is the SARS-CoV-2 genome length $(\mathrm{G}=29903)$. The means of each group are

617 presented by vertical dashed lines and reported in the figure (+/- 2SEM). e, Proportion of

618 observed nucleotide changes. Dashed lines indicate the expectation given the genome

619 composition under equal mutation probability for each type of nucleotide change. Vertical

620 bars in panels $\mathbf{c}$ and $\mathbf{e}$ represent the $95 \%$ confidence interval computed as $p \pm$

$621 \quad z \sqrt{\frac{p(1-p)}{N}}, z=1.96$.

622 
a

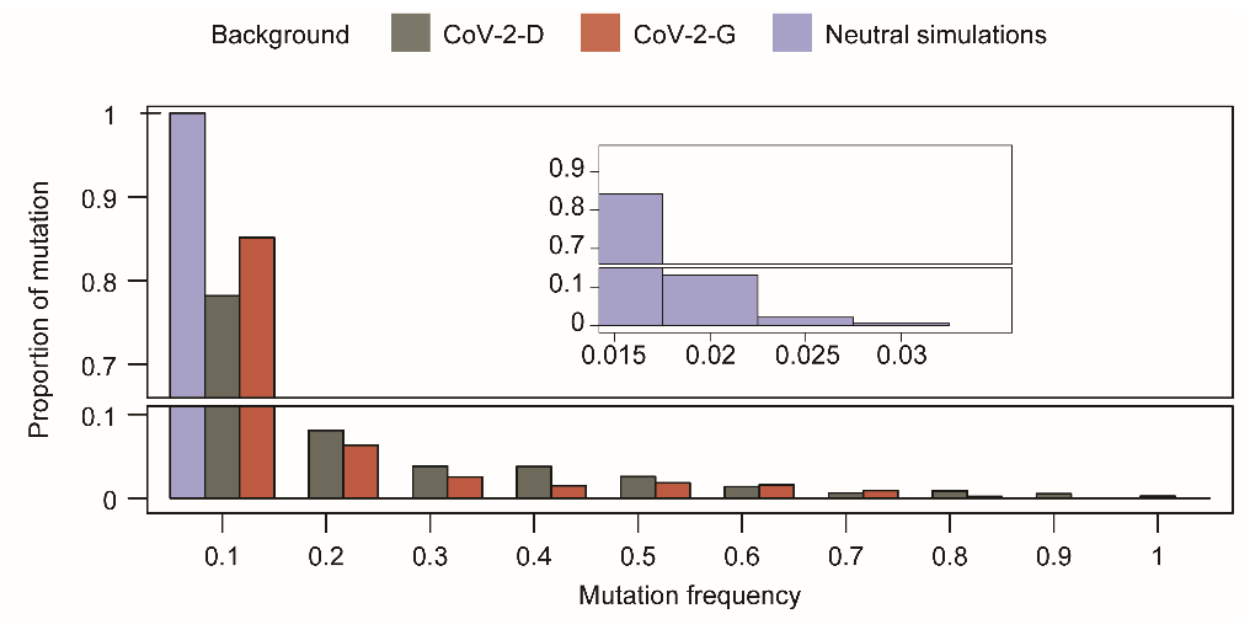

b

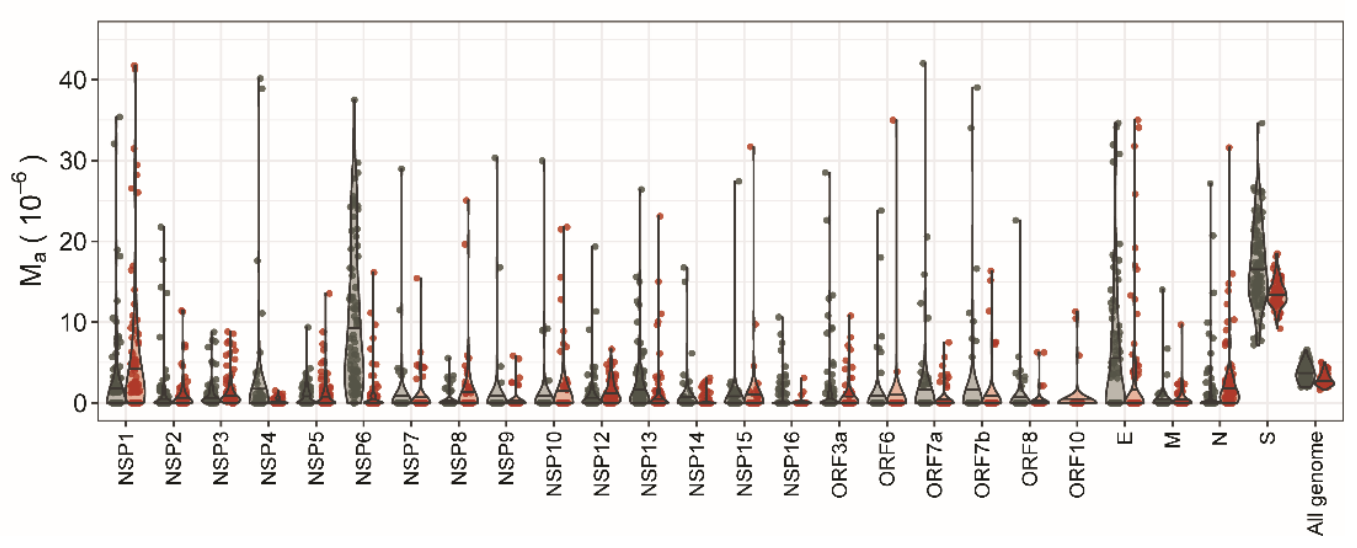

623

624

625

626

627

628

629

630

631

632

633

Fig. 2| Site frequency spectrum and heterogeneity across genes. a, Proportion of

mutations with a given frequency after 15 cycles of propagation in the CoV-2-D and

CoV-2-G genetic background or under a simulated neutral model of mutation

accumulation. The bump observed at high frequencies in the data is not compatible with the expectation of the neutral model. $\mathbf{b}$, Per-base mutation accumulation $\left(M_{a}\right)$ computed for each gene and for the entire genome shows heterogeneity. The spike gene has the largest accumulation rate in both backgrounds $\left(M_{a}^{(S)}=17.1 \pm 1.0,13.5 \pm 0.4 \cdot 10^{-6}\right.$, for the CoV-2-D and CoV-2-G respectively), which is more than 4 times their genomic average. For resolution purposes, few outliers with $M_{a}$ above 45 are not shown (see full set in Fig. S4). 


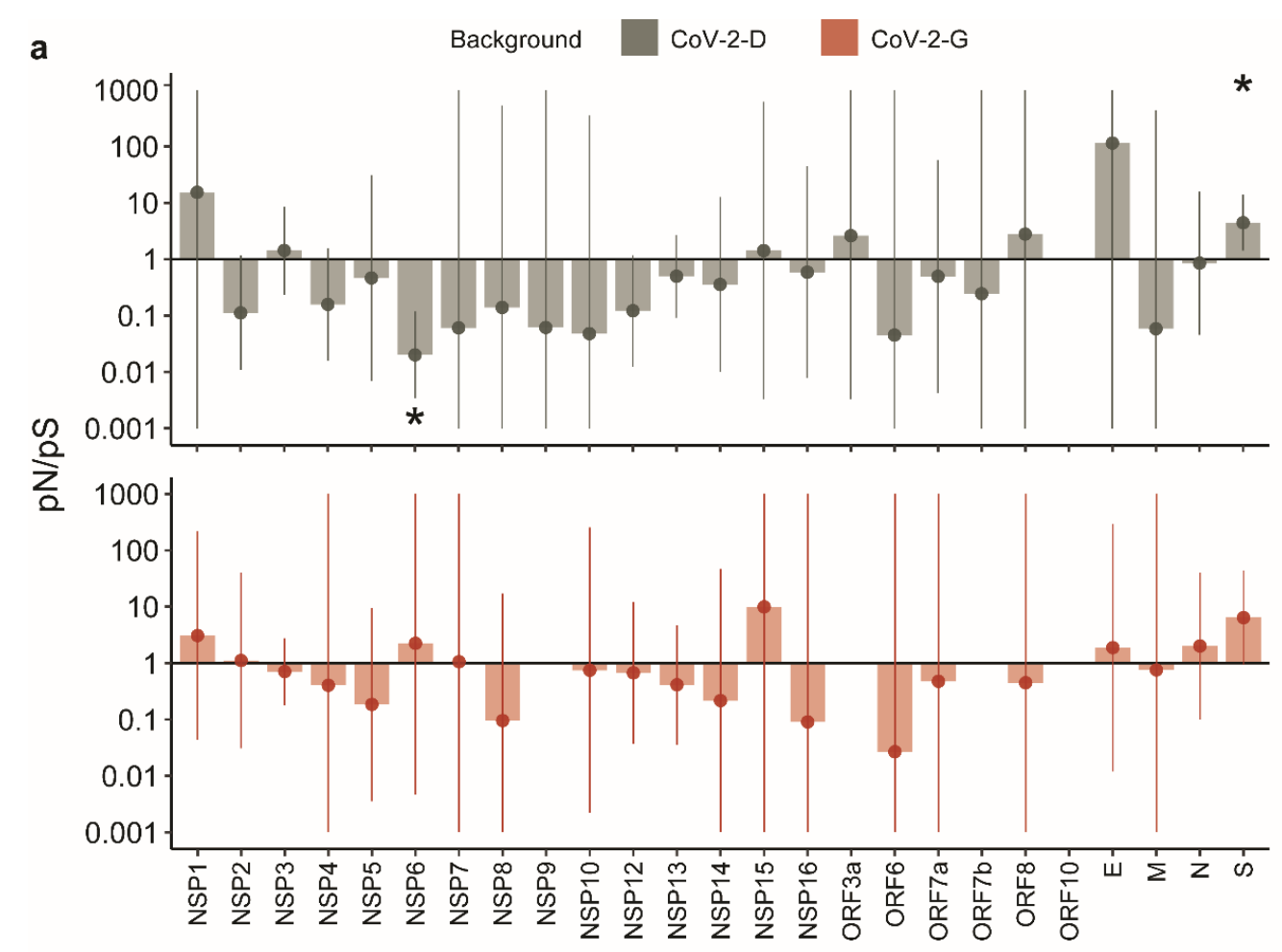

b

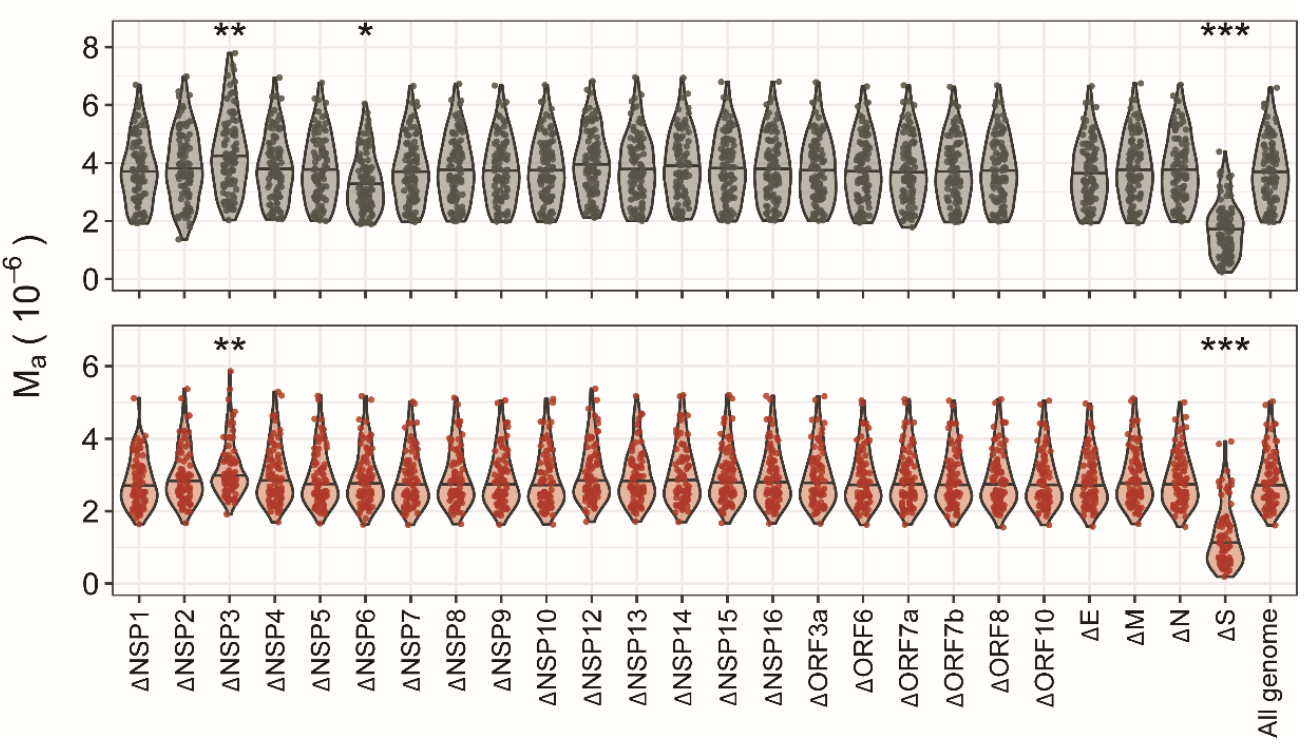

635 Fig. 3| Gene-wise signs of selection. a, The relative proportion of non-synonymous to

636 synonymous polymorphism, $p N / p S$, was computed for each gene and genetic

637 background (see Methodology). The horizontal line indicates the expectation under

638 neutrality $(\mathrm{pN} / \mathrm{pS}=1)$, values above suggest positive selection while values below

639 suggest purifying selection. Vertical bars show the $95 \%$ confidence intervals and the

640 stars indicate the genes where such interval does not include 1. For the sake of

641 resolution, we show the confidence intervals within the $\left[10^{-3}, 10^{3}\right]$ range. $\mathbf{b}$, Identifying

642 the genes that affect the estimation of mutation rate. Per-base mutation accumulation 
$643\left(M_{a}\right)$ was computed for the entire genome or by excluding each gene on at the time (e.g.

$644 \Delta$ ). The stars indicate the cases where removing the gene leads to an estimation of $M_{a}$ 645 significantly different from the all genome (non-parametric Wilcox test, p-value $<0.05$ $646\left(^{*}\right), 0.01\left(^{* *}\right)$ or $\left.0.001\left(^{* * *}\right)\right)$.

647

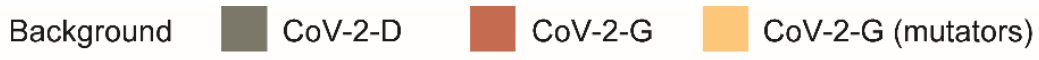

a

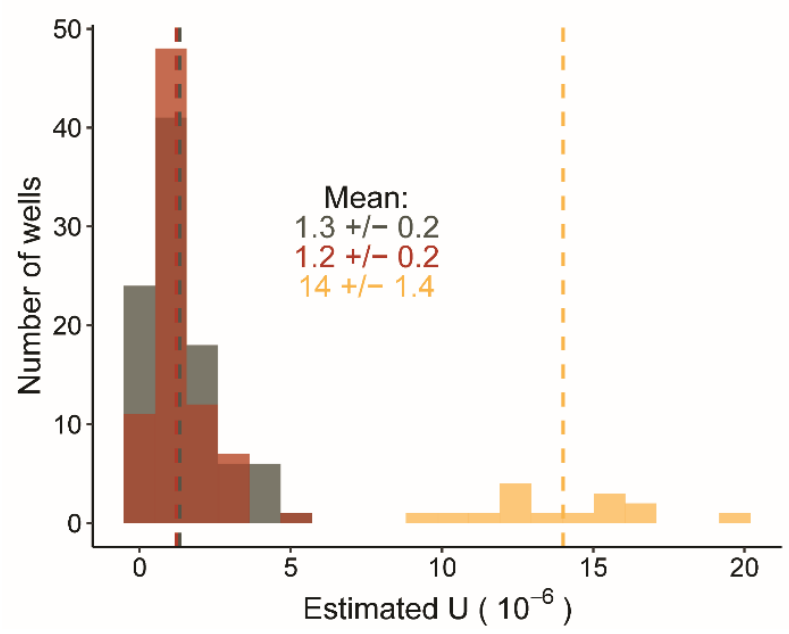

b

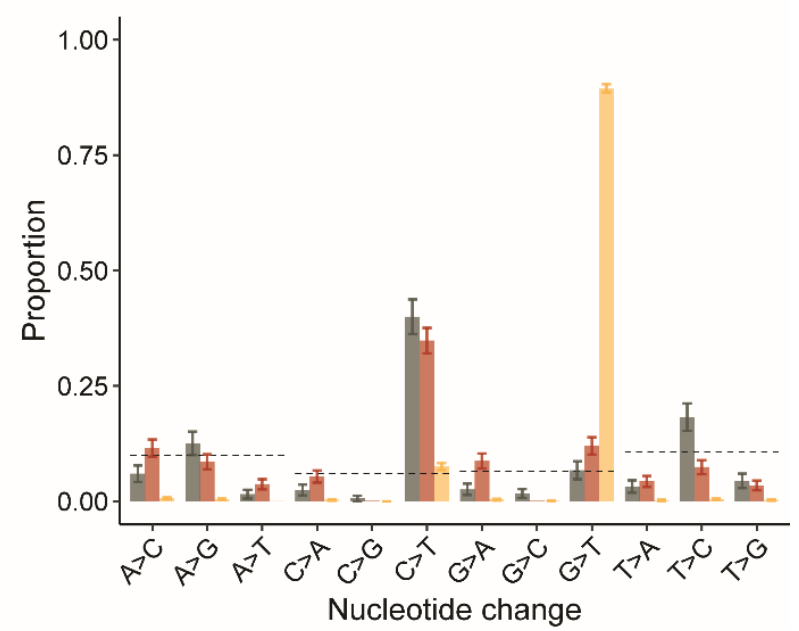

648

Fig. 4 | Estimation of mutation rates and bias excluding outlier genes. a, The per-

650 base per-infection cycle mutation rate was calculated by summing the observed

651 mutation frequencies as: $U=\frac{\sum f}{P * G}$, where $P$ is the number of passages $(P=15)$ and $\mathrm{G}$ is the

652 length of SARS-CoV-2 genome excluding the Nsp3, Nsp6 and Spike genes (29903-5835-

653 870-3822=19376). The means of each group are presented as vertical dashed lines and

654 reported in the figure (+/- 2SEM). b, Proportion of nucleotide changes observed

655 excluding the Nsp3, Nsp6 and Spike genes. Dotted lines indicate the expectation given

656 the genome composition under equal mutation probability for each type of nucleotide

657 change. Vertical bars represent the 95\% confidence interval computed as $p \pm$

658

$z \sqrt{\frac{p(1-p)}{N}}, z=1.96$

659 
a

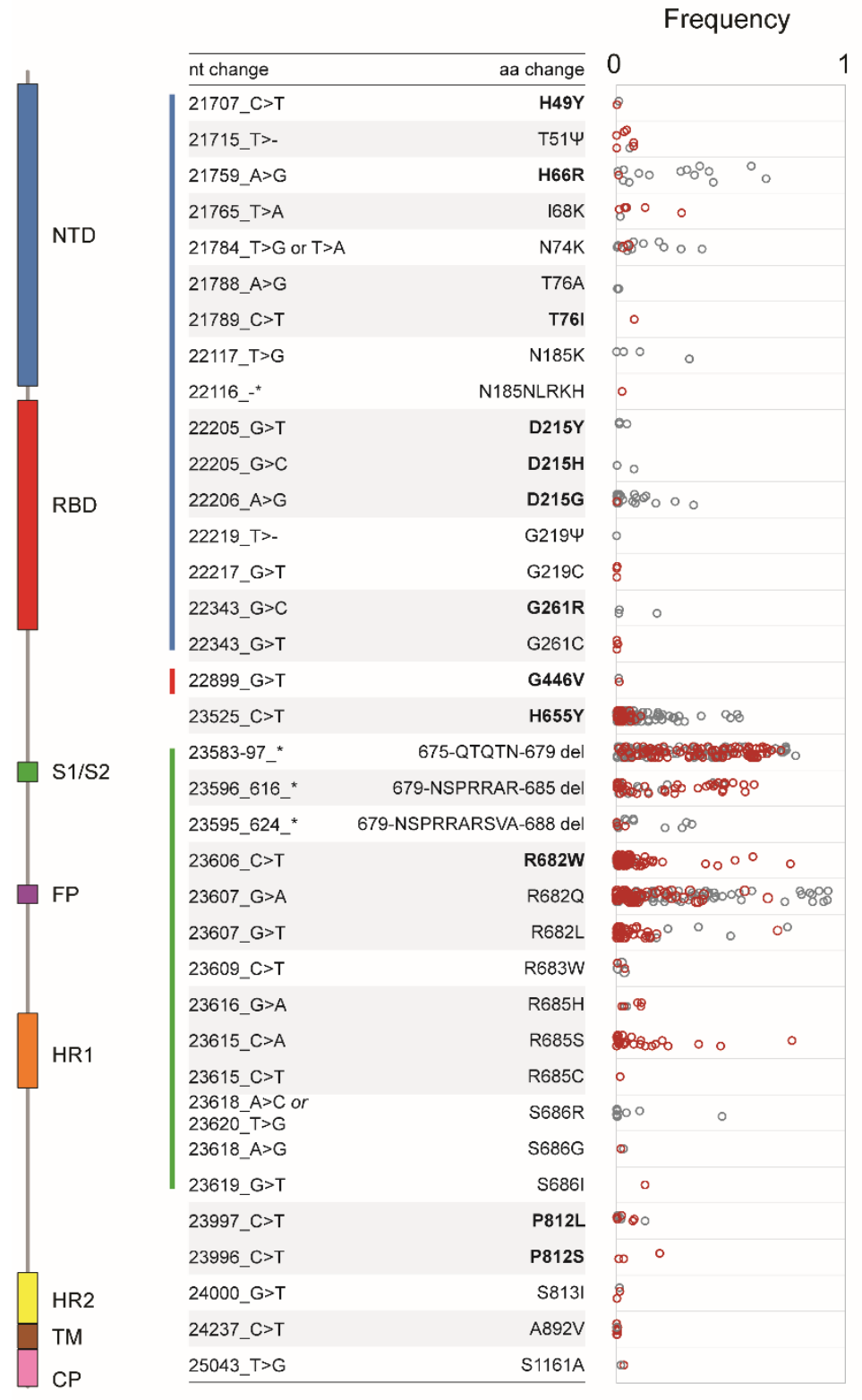

b

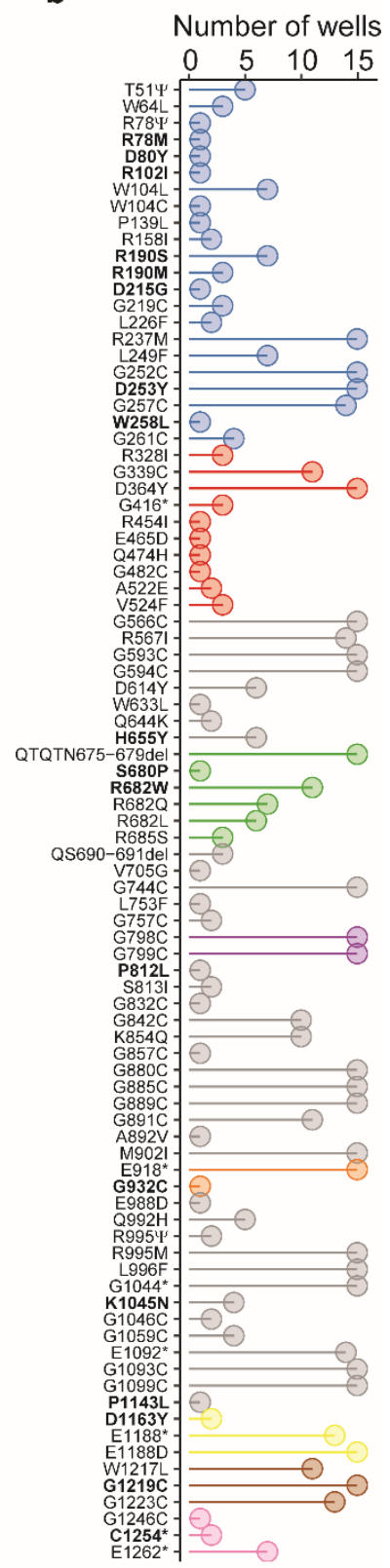

661 Fig. 5| Convergent evolution in the Spike gene. a, Mutations on S observed in both

662 CoV-2-D and CoV-2-G backgrounds and their frequencies in each well (open circles). b,

663 Non-synonymous mutations on the spike detected in the populations where the

664 mutators were observed (number of wells on the X-axis). The color annotation

665 represents the N-terminal domain (NTD, 14-305), the receptor-binding domain (RBD,

666 319-541), the cleavage site (S1/S2, 669-688), the fusion peptide (FP, 788-806), the

667 heptapeptide repeat sequences (HR1, 912-984 and HR2, 1163-1213), the TM domain

668 (1213-1237), and cytoplasm domain (CP, 1237-1273). Amino acids changes in bold

669 were also observed in the human population (as of 24 October 2021;

670 https://nextstrain.org/ncov/gisaid/global) (full list in Supplementary Table 6). 